\title{
Genome-wide association study of type 2 diabetes in Africa
}

\author{
Ji Chen ${ }^{1} \cdot$ Meng Sun ${ }^{2} \cdot$ Adebowale Adeyemo $^{3}$ - Fraser Pirie ${ }^{4} \cdot$ Tommy Carstensen $^{1,5} \cdot$ Cristina Pomilla $^{1,5}$. \\ Ayo P. Doumatey ${ }^{3}$. Guanjie Chen ${ }^{3}$. Elizabeth H. Young ${ }^{1,5}$. Manjinder Sandhu ${ }^{1,5}$. Andrew P. Morris ${ }^{2,6}$. \\ Inês Barroso ${ }^{1,7} \cdot$ Mark I. McCarthy $^{2,8,9} \cdot$ Anubha Mahajan $^{2}$ • Eleanor Wheeler ${ }^{1,7}$. Charles N. Rotimi ${ }^{3}$. Ayesha A. Motala ${ }^{4}$
}

Received: 12 September 2018 / Accepted: 22 March 2019 / Published online: 2 May 2019

(C) The Author(s) 2019

\begin{abstract}
Aims/hypothesis Genome-wide association studies (GWAS) for type 2 diabetes have uncovered $>400$ risk loci, primarily in populations of European and Asian ancestry. Here, we aimed to discover additional type 2 diabetes risk loci (including Africanspecific variants) and fine-map association signals by performing genetic analysis in African populations.

Methods We conducted two type 2 diabetes genome-wide association studies in 4347 Africans from South Africa, Nigeria, Ghana and Kenya and meta-analysed both studies together. Likely causal variants were identified using fine-mapping approaches.

Results The most significantly associated variants mapped to the widely replicated type 2 diabetes risk locus near TCF7L2 $(p=$ $5.3 \times 10^{-13}$ ). Fine-mapping of the TCF7L2 locus suggested one type 2 diabetes association signal shared between Europeans and Africans (indexed by rs7903146) and a distinct African-specific signal (indexed by rs17746147). We also detected one novel signal, rs73284431, near AGMO $\left(p=5.2 \times 10^{-9}\right.$, minor allele frequency [MAF] $=0.095$; monomorphic in most non-African populations), distinct from previously reported signals in the region. In analyses focused on 100 published type 2 diabetes risk loci, we identified 21 with shared causal variants in African and non-African populations.

Conclusions/interpretation These results demonstrate the value of performing GWAS in Africans, provide a resource to larger consortia for further discovery and fine-mapping and indicate that additional large-scale efforts in Africa are warranted to gain further insight in to the genetic architecture of type 2 diabetes.
\end{abstract}

Keywords Africa $\cdot$ Established loci $\cdot$ Fine-mapping $\cdot$ Genome-wide association study $\cdot$ Type 2 diabetes

$\overline{\text { Ji Chen, Meng Sun, Adebowale Adeyemo and Fraser Pirie contributed }}$ equally to this study.

Anubha Mahajan, Eleanor Wheeler, Charles N. Rotimi and Ayesha A. Motala are joint senior authors.

Electronic supplementary material The online version of this article (https://doi.org/10.1007/s00125-019-4880-7) contains peer-reviewed but unedited supplementary material, which is available to authorised users.

\author{
Ayesha A. Motala \\ motala@ukzn.ac.za \\ $\triangle$ Charles N. Rotimi \\ rotimic@mail.nih.gov \\ $\triangle$ Eleanor Wheeler \\ eleanor.wheeler@mrc-epid.cam.ac.uk \\ $\triangle$ Anubha Mahajan \\ anubha@well.ox.ac.uk
}

1 Wellcome Sanger Institute, Hinxton, Cambridge, UK

2 Wellcome Centre for Human Genetics, University of Oxford, Roosevelt Drive, Oxford OX3 7BN, UK
3 Center for Research on Genomics and Global Heath, National Human Genome Research Institute, National Institute of Health, Bethesda, MD, USA

4 Department of Diabetes and Endocrinology, University of KwaZulu-Natal, Durban 4013, South Africa

5 Department of Medicine, University of Cambridge, Cambridge, UK

6 Department of Biostatistics, University of Liverpool, Liverpool, UK

7 MRC Epidemiology Unit, University of Cambridge School of Clinical Medicine, Institute of Metabolic Science, Cambridge Biomedical Campus, Cambridge CB2 0QQ, UK

8 Oxford Centre for Diabetes, Endocrinology and Metabolism, University of Oxford, Oxford, UK

9 Oxford NIHR Biomedical Research Centre, Oxford, UK 


\section{Research in context}

\section{What is already known about this subject?}

- More than 400 type 2 diabetes risk loci have been found, primarily in populations of European and Asian ancestry

- Haplotype blocks in African populations are shorter than those in other ancestries, which may help when finemapping disease-associated loci

\section{What is the key question?}

- Can genetic studies in Africans help us gain further insights regarding the genetic architecture of type 2 diabetes, such as detecting novel risk loci and locating causal variants?

\section{What are the new findings?}

- We identified a novel association signal at $A G M O$ ( $r$ 73284431), which is distinct from previously reported signals in the region, and a second intergenic association signal at the well-established TCF $\angle 22$ locus. This second signal (indexed by rs 17746147) is distinct between European and African populations

- We identified 21 loci with shared causal variants in African and non-African populations

\section{How might this impact on clinical practice in the foreseeable future?}

- The use of genetic risk scores for individual stratification cannot be directly extrapolated from European ancestry populations to African populations; additional studies validating findings in Africans are therefore required

$\begin{array}{ll}\text { Abbreviations } \\ \text { AADM } & \text { Africa America Diabetes Mellitus } \\ \text { DCC } & \text { Durban Diabetes Case Control study } \\ \text { DDS } & \text { Durban Diabetes Study } \\ \text { GRS } & \text { Genetic risk score } \\ \text { GWAS } & \text { Genome-wide association study } \\ \text { LD } & \text { Linkage disequilibrium } \\ \text { MAF } & \text { Minor allele frequency } \\ \text { NIHR } & \text { National Institute for Health Research } \\ \text { PC } & \text { Principal component } \\ \text { QC } & \text { Quality control } \\ \text { VNTR } & \text { Variable number tandem repeat }\end{array}$

\section{Introduction}

Type 2 diabetes is a major and growing public health problem, with Africa being the region with the fastest growing prevalence [1-3]. In addition to lifestyle, genetic factors play a major role in susceptibility to type 2 diabetes. To date, type 2 diabetes genome-wide association studies (GWAS) have uncovered over 400 risk signals, primarily in populations of European $[4,5]$ and Asian [6-8] ancestry, with more limited efforts in Hispanics/Latinos [9-11] and African-Americans [12, 13]. Thus far, type 2 diabetes genetic studies in populations from Africa, which are genetically and environmentally diverse, have focused on the replication of established loci [14].

Here, we conducted a meta-analysis of type 2 diabetes in up to 4347 African participants to identify genetic risk factors associated with type 2 diabetes in Africans, evaluate previously reported loci and utilise the finer-grained linkage disequilibrium (LD) pattern of African populations to finemap-associated loci.

\section{Methods}

Study participants South African Zulu individuals were type 2 diabetes cases and controls from two studies: the Durban Diabetes Study (DDS) and the Durban Diabetes Case Control study (DCC). DDS was a population-based cross-sectional study of non-pregnant urban black African adults of Zulu descent, aged $>18$ years, residing in Durban, South Africa $[15,16]$. Further details are provided in the electronic supplementary material (ESM) Methods. Additional type 2 diabetes cases from the same ethnic group and locality were obtained from the DCC, which included individuals with type 2 diabetes attending a diabetes clinic. Type 2 diabetes was defined using WHO criteria $[15,16]$. The combined type 2 diabetes cases and controls from DDS and DCC were aggregated into a single Zulu study.

The Africa America Diabetes Mellitus (AADM) study comprised individuals from sub-Saharan Africa, enrolled from university medical centres in Nigeria, Ghana and Kenya. A person with type 2 diabetes was identified using ADA criteria or if he or she was receiving treatment for type 2 diabetes. Probable cases of type 1 diabetes were excluded and controls had no evidence of diabetes based on fasting $/ 2 \mathrm{~h}$ glucose or symptoms of suggestive diabetes [14]. The characteristics of the Zulu and AADM participants are shown in ESM Table 1. 
Genotyping, quality control and imputation In total, 2707 African individuals of Zulu descent (2003 women and 704 men) were genotyped using the Illumina Multi-Ethnic Genotyping Array (Illumina, Illumina Way, San Diego, CA, USA). Following sample and variant quality control (QC) (ESM Methods), there were 2578 samples with genotype and phenotype information (1602 cases and 976 controls) and $1,434,868$ variants $(1,395,345$ autosomal and 39,523 on the $\mathrm{X}$ chromosome).

The AADM samples were genotyped on the Affymetrix Axiom PANAFR SNP array as described previously [14]. After QC (ESM Methods), there were 1031 cases and 738 controls and 2,141,465 variants (2,080,378 autosomal and 61,087 on the $\mathrm{X}$ chromosome) in AADM.

All samples were imputed to a merged panel of 1000 Genomes phase 3 [17] and African samples using IMPUTE2 [18] (Zulu) or positional Burrows-Wheeler transform (PBWT) [19] using the Sanger imputation server (AADM) (ESM Methods). We retained all imputed SNPs with MAF $>0.01$ and imputation information score $>0.4$ that were also in a newer version of the imputation panel.

Association analysis In Zulu samples, association with type 2 diabetes was performed for each variant based on the imputation dosage using a linear mixed model that accounts for the presence of related individuals and any population structure implemented in genome-wide efficient mixed-model association (GEMMA) [20], adjusting for age, sex and BMI. The kinship matrix was estimated from directly genotyped autosomal variants with MAF $>0.01$. In AADM, association with type 2 diabetes was performed for each variant based on the imputation dosage using an additive logistic regression model implemented in SNPTEST v2.5.2 [21] (Oxford University, Oxford, UK www.well.ox.ac.uk/ gav/resources/snptest_v2. 5.2_linux_x86_64_dynamic.tgz) adjusting for age, sex, BMI and the first three principal components (PCs) to account for population structure. The number of PCs adjusted for in AADM was determined by testing for the number of PCs using the minimum average partial test [22]. The first three PCs were determined to be significant and thus were adjusted for in the association analyses.

Meta-analysis, signal selection and fine-mapping Meta-analysis of the Zulu and AADM summary statistics for shared variants was performed using a fixed-effects meta-analysis (weighted for effective sample size) in METAL [23] and we applied double genomic-control correction (ESM Methods). To identify distinct signals of association, we performed approximate conditional analyses using the joint model implemented in genome-wide complex trait analysis (GCTA) [24, $25]$ and variants with $p<2.5 \times 10^{-8}$ in the joint model were selected as signals with genome-wide significance [26]. To estimate meta-analysis ORs, we also performed an inverse- variance-weighted meta-analysis using an approximation of the allelic $\log _{e} \mathrm{OR}$ and variance from the linear model in the Zulu samples [27], and the $\log _{e} \mathrm{OR}$ estimates obtained directly from SNPTEST for the AADM samples.

We used FINEMAP [28] (C. Benner, University of Helsinki, Finland www.christianbenner.com/finemap_v1.1_x86_64.tgz) to identify likely causal variants within $500 \mathrm{~kb}$ either side of the most significant variant at the loci TCF7L2 and AGMO in the African meta-analysis and in Europeans [5] (ESM Methods).

Comparison with established loci We used 'direct' (same lead variant with $p<0.05$ and directionally consistent) and 'local' (locus-level) detection to explore the extent to which existing GWAS signals (almost all from non-African samples) were detected in the African GWAS (ESM Methods, ESM Table 2). We used two complementary approaches to test for enrichment of signals detected using the 'direct' approach: (1) a binomial test taking significance $(p<0.05)$ and direction of effect into account; and (2) enrichment of directly detected variants, accounting for the properties of the variants in GARFIELD [29] (ESM Methods). For loci demonstrating 'direct' and/or 'local' detection between our African data and existing GWAS signals (ESM Table 2), we performed co-localisation analyses implemented in the R package 'coloc' [30] using summary statistics from the largest available European type 2 diabetes GWAS at the time of analysis [5] (default prior for causal variant sharing set to 0.5) (ESM Methods). Using the weighted allele frequencies and sample sizes from the African meta-analysis and previously reported effect sizes, we estimated the power to detect established variants at the significance thresholds $p<0.05$ and $p<2.5 \times 10^{-8}$ using R version 3.3.0 [31] (ESM Table 2).

We also performed genetic risk score (GRS) analyses to harvest association information from multiple variants. GRSs were calculated as the total number of risk alleles in subsets of the 102 variants at established loci from existing GWAS studies of type 2 diabetes (published before May 2018), primarily in populations of European and Asian ancestry (ESM Table 2).

Testing association of INS-variable number tandem repeat with type 2 diabetes We used the haplotypic information for INS-variable number tandem repeat (VNTR) generated in African-descent individuals by Stead et al (2003) [32] to impute INS-VNTR lineages in the Zulu and AADM samples and perform a meta-analysis (ESM Methods). Conditional analysis was performed to detect distinct association signals by inclusion of dosages of the lead type 2 diabetes variants as covariates in the regression model.

Ethics statements Ethical approval was obtained for each participating cohort: the Institutional Review Board for each AADM participating institution and the Biomedical 
Research Ethics Committee of the University of KwaZuluNatal for DCC (BF078/08) and DDS (BF030/12). DDS also had UK National Research Ethics Service approval (reference: 14/WM/1061). Written informed consent was obtained from all participants and the study was conducted in accordance with the principles of the Declaration of Helsinki.

\section{Results}

A total of 12,148,595 variants (genotyped or imputed) overlapping the Zulu and AADM samples were included in the meta-analysis of type 2 diabetes. We identified two genomewide-significant $\left(p<2.5 \times 10^{-8}\right)[26]$ association signals (Table 1, Fig. 1). A further 37 distinct signals were detected at $2.5 \times 10^{-8} \leq p<1 \times 10^{-5}$ (ESM Table 3 ).

The most significant signal for type 2 diabetes association, rs7903146 at TCF7L2 $\left(p=5.3 \times 10^{-13}\right)$, has been widely reported in other ethnic groups $[9,13,33,34]$. The second signal, rs73284431, $\left(p=5.2 \times 10^{-9}\right.$, risk allele frequency $=0.093$, specific to individuals of African descent) maps to an intron of $A G M O, 491 \mathrm{~kb}$ upstream of the $D G K B$ transcription start site. The region is already known to harbour two independent GWAS signals for type 2 diabetes [5] and fasting glucose [35, 36] (denoted by rs 10238625 and rs $10276674,132 \mathrm{~kb}$ apart and $>379 \mathrm{~kb}$ from rs73284431), both of them distinct from our lead variant based on exact conditional analyses (ESM Table 4).

Despite not reaching genome-wide significance, the association with $\mathrm{rs} 12277475\left(p=2.0 \times 10^{-7}\right.$, ESM Table 3) near the INS (insulin) gene was of interest given previous reports of a significant African-American type 2 diabetes association signal at rs 3842770 [37], $28 \mathrm{~kb}$ away. The two lead variants are not in $\operatorname{LD}\left(r^{2} \sim 0.03\right)$ in 2959 African samples from the merged imputation reference panel [13], and reciprocal exact conditional analyses confirmed our signal was not being driven by the reported African-American association signal (ESM Table 5). In our data, rs3842770 showed a more modest association with type 2 diabetes ( $p=0.0020$ in the joint meta-analysis) than rs12277475. The rs 12277475 association was also not driven by the previously reported East Asian type 2 diabetes signal (rs7107784) in this region [7] (ESM Table 5).

In type 1 diabetes, robust associations with variants at INS$I G F 2$ have been localised to the INS-VNTR mini-satellite within the INS gene promoter [38, 39]; in Europeans, an association between VNTR class III alleles and type 2 diabetes predisposition has been reported in historical candidate gene studies $[40,41]$. To explore the relationship between the rs 12277475 signal and VNTR variation, we imputed INSVNTR genotypes using flanking SNP haplotypes (see Methods and ESM Methods). Of the nine distinct lineages with MAF $>0.01$ detected in both Zulu and AADM, the strongest associations were observed for lineage $\mathrm{W}(p=0.0001$, OR 1.24, ESM Table 6) and lineage $\mathrm{K}(p=0.0057$, OR
0.85 , ESM Table 6). Type 2 diabetes association results for lineages $\mathrm{W}$ and $\mathrm{K}$ and lead variant rs12277475 were largely unchanged in reciprocal conditional analyses (ESM Tables 7 and 8 [Zulu and AADM, respectively]). We conclude that rs12277475 is likely to represent a novel type 2 diabetes association in this region, independent of previous genome-wide significant associations detected in the close vicinity, and is not acting through the VNTR.

Fine-mapping of TCF7L2 and AGMO Fine-mapping of TCF7L2 and AGMO identified the most significant variants from the meta-analysis, rs7903146 and rs73284431, as the most likely causal variants with posterior probabilities of association of 0.996 and 0.828 , respectively (ESM Table 9). These were the only SNPs in the top configuration of causal variants from FINEMAP (ESM Figs. 1 and 2). At TCF7L2, a second plausible causal variant, rs17746147 $\left(r^{2}=0.009\right.$ with rs7903146 estimated from the African samples in the merged panel), with posterior probability of association of 0.295 , was contained in the second most likely configuration of causal variants (ESM Table 9, ESM Fig. 1). The 99\% credible intervals and corresponding results in Europeans are presented in ESM Table 9. Fine-mapping results were comparable when using the stepwise approximate Bayes factor approach [42] (data not shown).

Detection of established loci We explored the extent to which previously reported type 2 diabetes association signals could be detected in African-descent individuals. Based on the previously reported effect sizes and the effect allele frequency and sample size from our African meta-analysis, we had sufficient power $(80 \%)$ to detect three signals (TCF7L2, DNER and SRR) at genome-wide significance $\left(p<2.5 \times 10^{-8}\right)$ (ESM Table 2). Only the $T C F 7 L 2$ variant reached genome-wide significance in our study, whereas both variants in DNER (rs1861612) and $S R R$ ( $\mathrm{rs} 391300$ ), originally discovered in Pima Indians and East Asians, respectively, had $p>0.1$ (ESM Table 2).

So far, five African-American type 2 diabetes-associated signals have been reported, three of which (two in $K C N Q 1$ and one in $H M G A 2$ ) were first reported in Europeans and two (INS-IGF2 and HLA-B) were first reported in AfricanAmericans (ESM Table 2) [13]. In our meta-analysis, we detected only a nominal association for the African-American INS-IGF2 signal ( $\mathrm{rs} 3842770, p=0.0020$, ESM Table 2). However, we identified another signal at this locus (rs12277475, $p=2.0 \times 10^{-7}$, ESM Table 3) that is independent of the African-American signal (rs3842770), and this signal co-localises with association in Europeans (posterior probability $\mathrm{H} 4=1$, ESM Table 10).

In 'direct' analyses (same lead variant with $p<0.05$ and directionally consistent in the African meta-analysis), we detected 12 of 100 lead variants (ESM Table 2) at established type 2 diabetes loci first reported in non-African ancestry individuals, significantly more than expected by chance (binomial test 


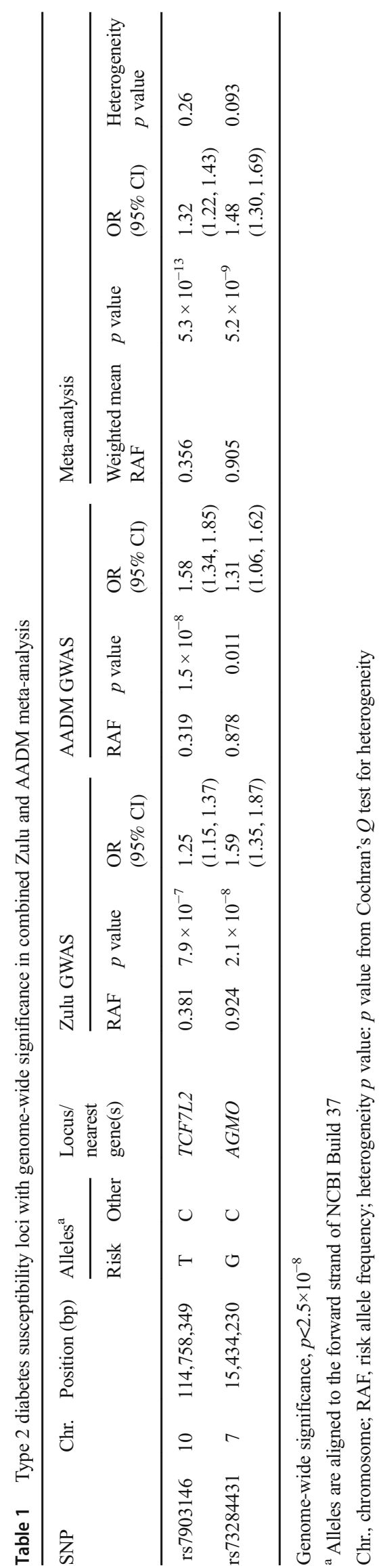




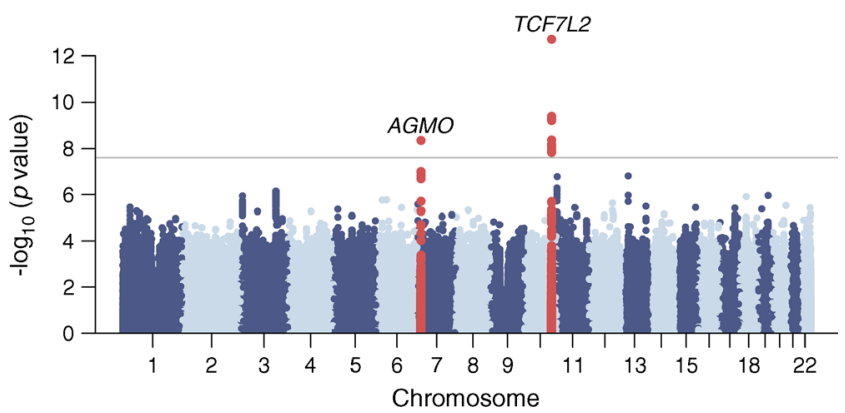

Fig. 1 Manhattan plot of the type 2 diabetes meta-analysis results. The horizontal grey line corresponds to $p=2.5 \times 10^{-8}$ and loci reaching that significance threshold (variants within $500 \mathrm{~kb}$ distance of those with $p<2.5 \times 10^{-8}$ ) are shown in red. Gene labels correspond to the nearest/most biologically plausible gene

of enrichment $p=8.14 \times 10^{-6}$, GARFIELD enrichment OR [95\% CI] 3.07 (1.50, 6.28), $p=0.002)$ (ESM Methods). In addition, detection of 'local' signals (at least one variant within the $200 \mathrm{~kb}$ region flanking the previously reported index variant [100 $\mathrm{kb}$ either side] reaching nominal significance $[p<0.05]$ after correcting for the effective number of independent tests) identified 11 type 2 diabetes loci, two of them overlapping signals also detected with the direct approach (ESM Table 2). Genetic co-localisation analyses suggested that African and non-African populations share the same causal variants at all
21 loci showing direct or local detection in our data (posterior probability $\mathrm{H} 4>0.8$, ESM Table 10 ).

We constructed GRSs by combining subsets of 102 previously established loci (including those first reported in Africanancestry individuals) and tested for association with type 2 diabetes (ESM Table 2). A GRS constructed from these variants showed significant association with type 2 diabetes in the Zulu (OR 1.05 per risk allele, $p=1.3 \times 10^{-5}$, Fig. 2a) and AADM samples (OR 1.02 per risk allele, $p=0.020$, Fig. $2 b$ ), an association driven primarily by the 13 directly detected variants (Zulu, OR 1.17, $p=1.7 \times 10^{-9}$; AADM, OR 1.05, $p=0.029$ ). GRSs based on the variants detected by only the local approach or the variants not detected by either approach were not significantly associated with type 2 diabetes in African samples $(p=$ 0.19 and 0.084 , respectively in the Zulu; $p=0.11$ and 0.32 , respectively in AADM) (Fig. 2). These results show there is a shared genetic contribution to type 2 diabetes at established loci directly detected in Africans.

\section{Discussion}

In a meta-analysis of type 2 diabetes from two African populations, we replicated the widely reported association at
Fig. 2 (a) Associations between the GRSs constructed from the subsets of established type 2 diabetes variants and type 2 diabetes in the Zulu samples. (b) Associations between the GRSs constructed from the subsets of established type 2 diabetes variants and type 2 diabetes in the AADM samples. Please see ESM Table 2 for details of categories a

\begin{tabular}{lc}
\multicolumn{1}{c}{ Category } & No. of variants \\
Directly detected & 13 \\
Locally detected only & 9 \\
All detected & 22 \\
Not detected & 77 \\
All except directly detected & 86 \\
All loci & 99
\end{tabular}

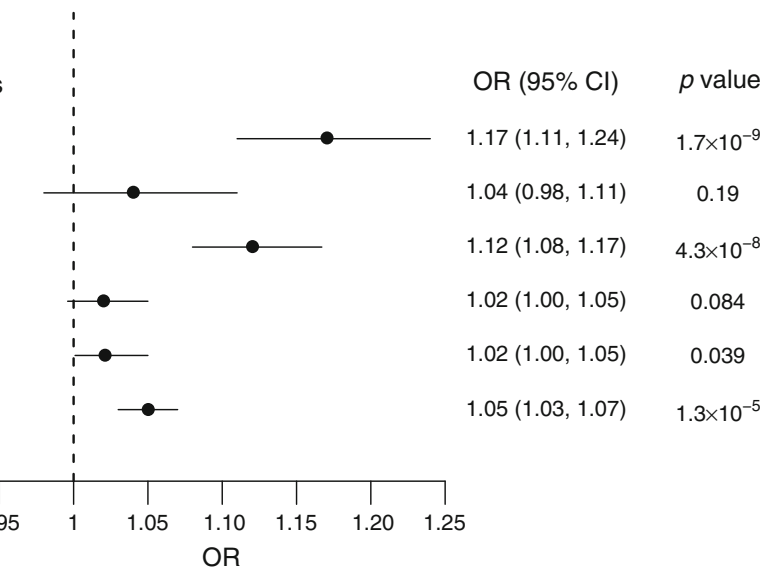

b

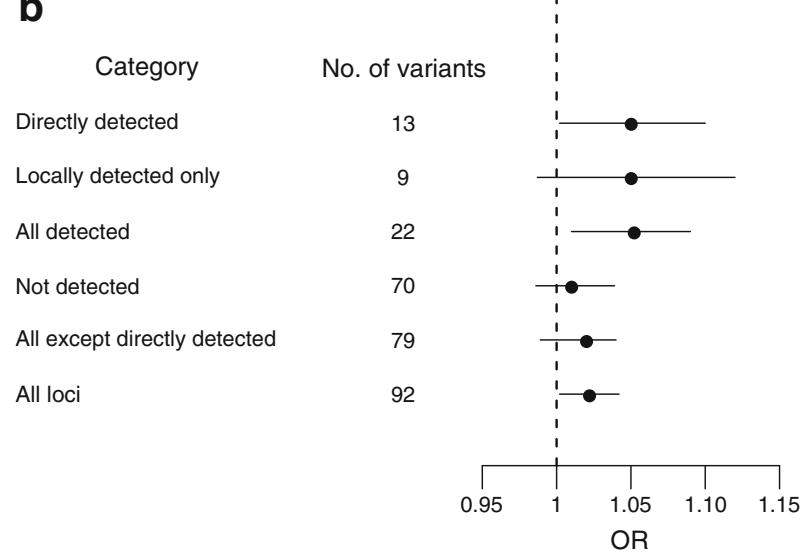

OR $(95 \% \mathrm{Cl}) \quad p$ value

$1.05(1.00,1.10) \quad 0.029$

$1.05(0.99,1.12) \quad 0.11$

$1.05(1.01,1.09) \quad 5.8 \times 10^{-3}$

$1.01(0.99,1.04) \quad 0.32$

$1.02(0.99,1.04) \quad 0.11$

$1.02(1.00,1.04)-0.020$ 
TCF7L2 (rs7903146) and identified a novel association signal at $A G M O$ (rs 73284431) that is distinct from previously reported signals in the region.

Using direct and local detection, we showed the transferability of 21 established type 2 diabetes signals discovered in non-African ancestry populations to Africans and that causal variants at those loci were shared across ancestries. For example, the lead SNP at TCF7L2, rs7903146, shared between African and European ancestries, has been refined as the causal variant by examining LD blocks of West African, Danish, Icelandic and American-African populations [43, 44]. Although the mechanisms through which TCF7L2 variation increases type 2 diabetes risk are largely unknown, recent evidence implicates altered incretin signalling [45].

We also found evidence for ancestry-specific signals, such as the second intergenic association signal at TCF $7 L 2$ that is distinct between European and African populations (indexed by rs17746147). In addition, although not reaching genomewide significance, we detected an association at the INS-IGF2 locus (rs12277475) that appears to be distinct from the previously reported signals in African-Americans and East Asians in the region. We found no evidence of association between the lead variant and VNTR variation in our study, suggesting that the association at INS-IGF2 in our data is not acting through the VNTR.

In summary, our findings highlight the importance of diverse ancestries for uncovering novel biology. Larger African meta-analyses are warranted to gain further insight on the genetic architecture of type 2 diabetes.

Acknowledgements This research has been conducted using the UK Biobank Resource (Application Number 9161).

Data availability Summary statistics from the meta-analysis will be available to download from the EBI GWAS Catalog (www.ebi.ac.uk/gwas/ downloads/summary-statistics).

Funding MIM is a Wellcome Senior Investigator and a National Institute for Health Research (NIHR) Senior Investigator. MIM acknowledges support for this work from Wellcome $(090532,203141,106130,098381$, 090367), MRC (G0601261) and NIH (U01DK105535, R01DK098032, U01DK085545). The DCC was funded by Servier South Africa, the South African Sugar Association and the Victor Daitz Foundation. CNR, AA, GC and APD are supported by the Intramural Research Program of the National Institutes of Health in the Center for Research on Genomics and Global Health (CRGGH). The CRGGH is supported by the National Human Genome Research Institute, the National Institute of Diabetes and Digestive and Kidney Diseases, the Center for Information Technology and the Office of the Director at the National Institutes of Health (1ZIAHG200362). IB acknowledges funding from Wellcome (WT206194). The study sponsors were not involved in the design of the study; the collection, analysis, and interpretation of data; writing the report; or the decision to submit the report for publication.

Duality of interest TTC owns shares in Novo Nordisk, Roche and Teva Pharmaceutical Industries. IB and spouse own stock in GlaxoSmithKline and Incyte Corporation. MIM serves on advisory panels for Pfizer, Novo Nordisk and Zoe Global; has received honoraria from Pfizer, Novo Nordisk and Lilly; has stock options in Zoe Global; and has received research funding from AbbVie, AstraZeneca, Boehringer Ingelheim, Lilly, Janssen, Merck, Novo Nordisk, Pfizer, Roche, Sanofi Aventis, Servier and Takeda. The views expressed in this article are those of the author(s) and not necessarily those of the NHS, the NIHR or the Department of Health.

Contribution statement JC, MeS, AA, FP, IB, MIM, AM, EW, CNR and AAM wrote the manuscript. JC, MeS, AA, TC, GC, APM, IB, MIM, AM and EW contributed to the analysis of the data. AA, FP, CP, APD, GC, AAM, EHY, MaS and CNR contributed to the acquisition of data. AAM, APD and CNR contributed to the phenotyping. FP, IB, MIM, CNR and AAM are the guarantors of this work and, as such, had full access to all the data in the study and take responsibility for the integrity of the data and the accuracy of the data analysis. All authors revised the manuscript critically for important intellectual content and approved the final version to be published.

Open Access This article is distributed under the terms of the Creative Commons Attribution 4.0 International License (http:// creativecommons.org/licenses/by/4.0/), which permits unrestricted use, distribution, and reproduction in any medium, provided you give appropriate credit to the original author(s) and the source, provide a link to the Creative Commons license, and indicate if changes were made.

\section{References}

1. NCD Risk Factor Collaboration (NCD-RisC) (2016) Worldwide trends in diabetes since 1980: a pooled analysis of 751 population-based studies with 4.4 million participants. Lancet 387:1513-1530

2. International Diabetes Federation (2015) Diabetes Atlas (7th Edition). Available from: http://www.diabetesatlas.org

3. Mbanya JC, Motala AA, Sobngwi E, Assah FK, Enoru ST (2010) Diabetes in sub-Saharan Africa. Lancet 375(9733):2254-2266. https://doi.org/10.1016/S0140-6736(10)60550-8

4. Morris AP, Voight BF, Teslovich TM et al (2012) Large-scale association analysis provides insights into the genetic architecture and pathophysiology of type 2 diabetes. Nat Genet 44:981-990

5. Scott RA, Scott LJ, Magi R et al (2017) An expanded genome-wide association study of type 2 diabetes in Europeans. Diabetes 66(11): 2888-2902. https://doi.org/10.2337/db16-1253

6. Kooner JS, Saleheen D, Sim X et al (2011) Genome-wide association study in individuals of South Asian ancestry identifies six new type 2 diabetes susceptibility loci. Nat Genet 43(10):984-989. https://doi.org/10.1038/ng.921

7. Imamura M, Takahashi A, Yamauchi $T$ et al (2016) Genome-wide association studies in the Japanese population identify seven novel loci for type 2 diabetes. Nat Commun 7(1):10531. https://doi.org/ 10.1038/ncomms 10531

8. Cho YS, Chen CH, Hu C et al (2011) Meta-analysis of genomewide association studies identifies eight new loci for type 2 diabetes in east Asians. Nat Genet 44:67-72

9. Qi Q, Stilp AM, Sofer T et al (2017) Genetics of type 2 diabetes in U.S. Hispanic/Latino individuals: results from the Hispanic Community Health Study/Study of Latinos (HCHS/SOL). Diabetes 66(5):1419-1425. https://doi.org/10.2337/db16-1150

10. Parra EJ, Below JE, Krithika S et al (2011) Genome-wide association study of type 2 diabetes in a sample from Mexico City and a meta-analysis of a Mexican-American sample from Starr County, Texas. Diabetologia 54(8):2038-2046. https://doi.org/10.1007/ s00125-011-2172-y

11. Consortium STD, Williams AL, Jacobs SB etal (2014) Sequence variants in SLC16A11 are a common risk factor for type 2 diabetes in Mexico. Nature 506(7486):97-101. https://doi.org/10.1038/nature12828 
12. Palmer ND, McDonough CW, Hicks PJ et al (2012) A genomewide association search for type 2 diabetes genes in African Americans. PLoS One 7(1):e29202. https://doi.org/10.1371/ journal.pone.0029202

13. Ng MCY, Shriner D, Chen BH et al (2014) Meta-analysis of genome-wide association studies in African Americans provides insights into the genetic architecture of type 2 diabetes. PLoS Genet 10(8). https://doi.org/10.1371/journal.pgen.1004517

14. Adeyemo AA, Tekola-Ayele F, Doumatey AP et al (2015) Evaluation of genome wide association study associated type 2 diabetes susceptibility loci in Sub Saharan Africans. Front Genet $6: 335$

15. Hird TR, Pirie FJ, Esterhuizen TM et al (2016) Burden of diabetes and first evidence for the utility of $\mathrm{HbA}_{1 \mathrm{c}}$ for diagnosis and detection of diabetes in urban black South Africans: The Durban Diabetes Study. PLoS One 11(8):e0161966. https://doi.org/10. 1371/journal.pone.0161966

16. Hird TR, Young EH, Pirie FJ et al (2016) Study profile: the Durban Diabetes Study (DDS): a platform for chronic disease research. Glob Health Epidemiol Genom 1:e2. https://doi.org/10.1017/ gheg.2015.3

17. Genomes Project C, Auton A, Brooks LD et al (2015) A global reference for human genetic variation. Nature 526:68-74

18. Howie BN, Donnelly P, Marchini J (2009) A flexible and accurate genotype imputation method for the next generation of genomewide association studies. PLoS Genet 5(6):e1000529. https://doi. org/10.1371/journal.pgen.1000529

19. Durbin R (2014) Efficient haplotype matching and storage using the positional Burrows-Wheeler transform (PBWT). Bioinformatics 30(9):1266-1272. https://doi.org/10.1093/ bioinformatics/btu014

20. Zhou X, Stephens M (2012) Genome-wide efficient mixed-model analysis for association studies. Nat Genet 44(7):821-824. https:// doi.org/10.1038/ng.2310

21. Marchini J, Howie B, Myers S, McVean G, Donnelly P (2007) A new multipoint method for genome-wide association studies by imputation of genotypes. Nat Genet 39(7):906-913. https://oi. org/10.1038/ng2088

22. Shriner D (2012) Improved eigenanalysis of discrete subpopulations and admixture using the minimum average partial test. Hum Hered 73(2):73-83. https://doi.org/10.1159/000335899

23. Willer CJ, Li Y, Abecasis GR (2010) METAL: fast and efficient meta-analysis of genomewide association scans. Bioinformatics 26(17):2190-2191. https://doi.org/10.1093/bioinformatics/btq340

24. Yang J, Lee SH, Goddard ME, Visscher PM (2011) GCTA: a tool for genome-wide complex trait analysis. Am J Hum Genet 88(1): 76-82. https://doi.org/10.1016/j.ajhg.2010.11.011

25. Yang J, Ferreira T, Morris AP et al (2012) Conditional and joint multiple-SNP analysis of GWAS summary statistics identifies additional variants influencing complex traits. Nat Genet 44(4):369375. https://doi.org/10.1038/ng.2213

26. Pe'er I, Yelensk R, Altshuler D, Daly MJ (2008) Estimation of the multiple testing burden for genomewide association studies of nearly all common variants. Genet Epidemiol 32(4):381-385. https:// doi.org/10.1002/gepi.20303

27. Cook JP, Mahajan A, Morris AP (2017) Guidance for the utility of linear models in meta-analysis of genetic association studies of binary phenotypes. Eur J Hum Genet 25(2):240-245. https://doi. org/10.1038/ejhg.2016.150

28. Benner C, Spencer CCA, Havulinna AS, Salomaa V, Ripatti S, Pirinen M (2016) FINEMAP: efficient variable selection using summary data from genome-wide association studies. Bioinformatics 32(10):1493-1501. https://doi.org/10.1093/ bioinformatics/btw018

29. Iotchkova V, Ritchie GRS, Geihs M et al (2019) GARFIELD classifies disease-relevant genomic features through integration of functional annotations with association signals. Nat Genet 51(2): 343-353. https://doi.org/10.1038/s41588-018-0322-6

30. Wallace C, Rotival M, Cooper JD et al (2012) Statistical colocalization of monocyte gene expression and genetic risk variants for type 1 diabetes. Hum Mol Genet 21(12):2815-2824. https://doi.org/10.1093/hmg/dds098

31. R Core Team (2016). R: A language and environment for statistical computing. R Foundation for Statistical Computing, Vienna, Austria. Available from www.R-project.org/ (accessed 11 May 2016)

32. Stead JD, Hurles ME, Jeffreys AJ (2003) Global haplotype diversity in the human insulin gene region. Genome Res 13(9):21012111. https://doi.org/10.1101/gr.948003

33. Takeuchi F, Serizawa M, Yamamoto K et al (2009) Confirmation of multiple risk loci and genetic impacts by a genome-wide association study of type 2 diabetes in the Japanese population. Diabetes 58(7):1690-1699. https://doi.org/10.2337/db08-1494

34. Tabassum R, Chauhan G, Dwivedi OP et al (2013) Genome-wide association study for type 2 diabetes in Indians identifies a new susceptibility locus at 2q21. Diabetes 62(3):977-986. https://doi. org $/ 10.2337 / \mathrm{db} 12-0406$

35. Dupuis J, Langenberg C, Prokopenko I et al (2010) New genetic loci implicated in fasting glucose homeostasis and their impact on type 2 diabetes risk. Nat Genet 42(2):105-132. https://doi.org/10. 1038/ng.520

36. Hwang JY, Sim X, Wu Y et al (2015) Genome-wide association meta-analysis identifies novel variants associated with fasting plasma glucose in East Asians. Diabetes 64(1):291-298. https://doi.org/ $10.2337 / \mathrm{db} 14-0563$

37. Ng MC (2015) Genetics of type 2 diabetes in African Americans. Curr Diab Rep 15(10):74. https://doi.org/10.1007/s11892-015-0651-0

38. Bennett ST, Wilson AJ, Esposito L et al (1997) Insulin VNTR allele-specific effect in type 1 diabetes depends on identity of untransmitted paternal allele. The IMDIAB group. Nat Genet 17(3):350-352. https://doi.org/10.1038/ng1197-350

39. Bell GI, Horita S, Karam JH (1984) A polymorphic locus near the human insulin gene is associated with insulin-dependent diabetes mellitus. Diabetes 33(2):176-183. https://doi.org/10.2337/diab.33. 2.176

40. Ahmed S, Bennett ST, Huxtable SJ, Todd JA, Matthews DR, Gough SC (1999) INS VNTR allelic variation and dynamic insulin secretion in healthy adult non-diabetic Caucasian subjects. Diabet Med 16(11): 910-917. https://doi.org/10.1046/j.1464-5491.1999.00169.x

41. Ong KK, Phillips DI, Fall C et al (1999) The insulin gene VNTR, type 2 diabetes and birth weight. Nat Genet 21(3):262-263. https:// doi.org/10.1038/6775

42. Wakefield J (2007) A Bayesian measure of the probability of false discovery in genetic epidemiology studies. Am J Hum Genet 81(2): 208-227. https://doi.org/10.1086/519024

43. Helgason A, Palsson S, Thorleifsson G et al (2007) Refining the impact of TCF7L2 gene variants on type 2 diabetes and adaptive evolution. Nat Genet 39(2):218-225. https://doi.org/10.1038/ng1960

44. Palmer ND, Hester JM, An SS et al (2011) Resequencing and analysis of variation in the TCF7L2 gene in African Americans suggests that SNP rs7903146 is the causal diabetes susceptibility variant. Diabetes 60(2):662-668. https://doi.org/10.2337/db10-0134

45. Srinivasan S, Kaur V, Chamarthi B et al (2018) TCF7L2 genetic variation augments incretin resistance and influences response to a sulfonylurea and metformin: the Study to Understand the Genetics of the Acute Response to Metformin and Glipizide in Humans (SUGAR-MGH). Diabetes Care 41(3):554-561. https://doi.org/ $10.2337 / \mathrm{dc} 17-1386$

Publisher's note Springer Nature remains neutral with regard to jurisdictional claims in published maps and institutional affiliations. 